\title{
Ion-selective optode microsensors based on hydrophobic solvatochromic dyes
}

\author{
Lu Wang ${ }^{1}$, Stephanie Sadler ${ }^{1}$, Eric Bakker* ${ }^{1}$ \\ ${ }^{1}$ Department of Inorganic and Analytical Chemistry, University of Geneva, Quai Ernest-Ansermet 30, \\ $\mathrm{CH}-1211$ Geneva, Switzerland, \\ Email: lu.wang@unige.ch
}

\begin{abstract}
:
Traditional ion-selective optodes based ion-exchange progress typically use a lipophilic $\mathrm{pH}$ indicator (also called chromoionophore) and give a cross response to sample pH changes, which has been very difficult to overcome until the introduction of electrically charged solvatochromic dyes (SDs). However, water soluble SDs are expelled to the aqueous phase during the ion-exchange progress, which is undesired. To solve this limitation, hydrophobic solvatochromic dyes are introduced. They localize the ion-exchange process for ion-selective optodes, rendering them $\mathrm{pH}$ independent while avoiding dye leakage. Structurally different lipophilized solvatochromic dyes are presented here for this purpose. Sensors containing SDs with a hydrophilic oxyethylene group are more sensitive and have lower detection limit compared to SDs with hydrophobic alkyl groups. Their response range can be successfully tuned by the choice of the dye so that they are potentially more attractive for on site, bioanalytical and imaging applications.
\end{abstract}

Key words: ion-selective optode, solvatochromic dyes, lipophilicity, response range

\section{Introduction}

A range of optical ion sensors have been discovered, including fluorescent molecular probes, surface modified nanomaterials, and biologically expressed proteins. Most metal selective optical molecular sensors are based on PET (photoinduced electron transfer) or ICT (intramolecular charge transfer) and are selective for transition metals by a strong ligand field effect. The selectivity of these sensors is not always predictable, making sensor design difficult. On the other hand, these types of sensors are normally single use owing to irreversibility of the coordination progress.

Ion-selective optical reversible sensors based on an equilibrium ion-exchange process have therefore been developed during the last few decades ${ }^{[1]}$. These optodes are composed of a polymeric phase containing a lipophilic optical reporter which is can be a $\mathrm{pH}$ indicator or, more recently, a positively charged solvatochromic dye (SD) ${ }^{[2]}$, an ion exchanger and an optically silent ionophore selective for the analyte. By exchanging the ions with the help of the optode components, the dynamic range and selectivity can easily be modified, and are not limited to a particular metal. Many recent fruitful directions have come from this approach, yielding sensor concepts that are more sensitive, operationally more reversible and more rapid.

Microsensors based on surface modified polystyrene microspheres were first proposed in our group ${ }^{[3]}$. By means of $\zeta$-potential, we further studied the mechanism of hydrophobic SDs participating in the ion-exchange progress. Lipophilic SDs remain anchored to the organic phase during the exchange process, and only the ionic chromophore functional group may be released into the aqueous phase and provide the optical response (Scheme 1). However, the adjustment of the hydrophobic properties of such SDs is a key approach to tune the response range effectively. We prepared here $\mathrm{K}^{+}$-selective PS microsensors modified with a range of different SDs that were synthesized in house. Sensors containing SDs with a hydrophilic oxyethylene group are more sensitive and exhibit a lower detection limit compared to SDs with a hydrophobic alkyl group. The calibration curve shifted to higher concentration with increasing lipophilicity of the SDs. Their response range can be successfully tuned by the choice of the dye so that they may meet much broader application needs. 

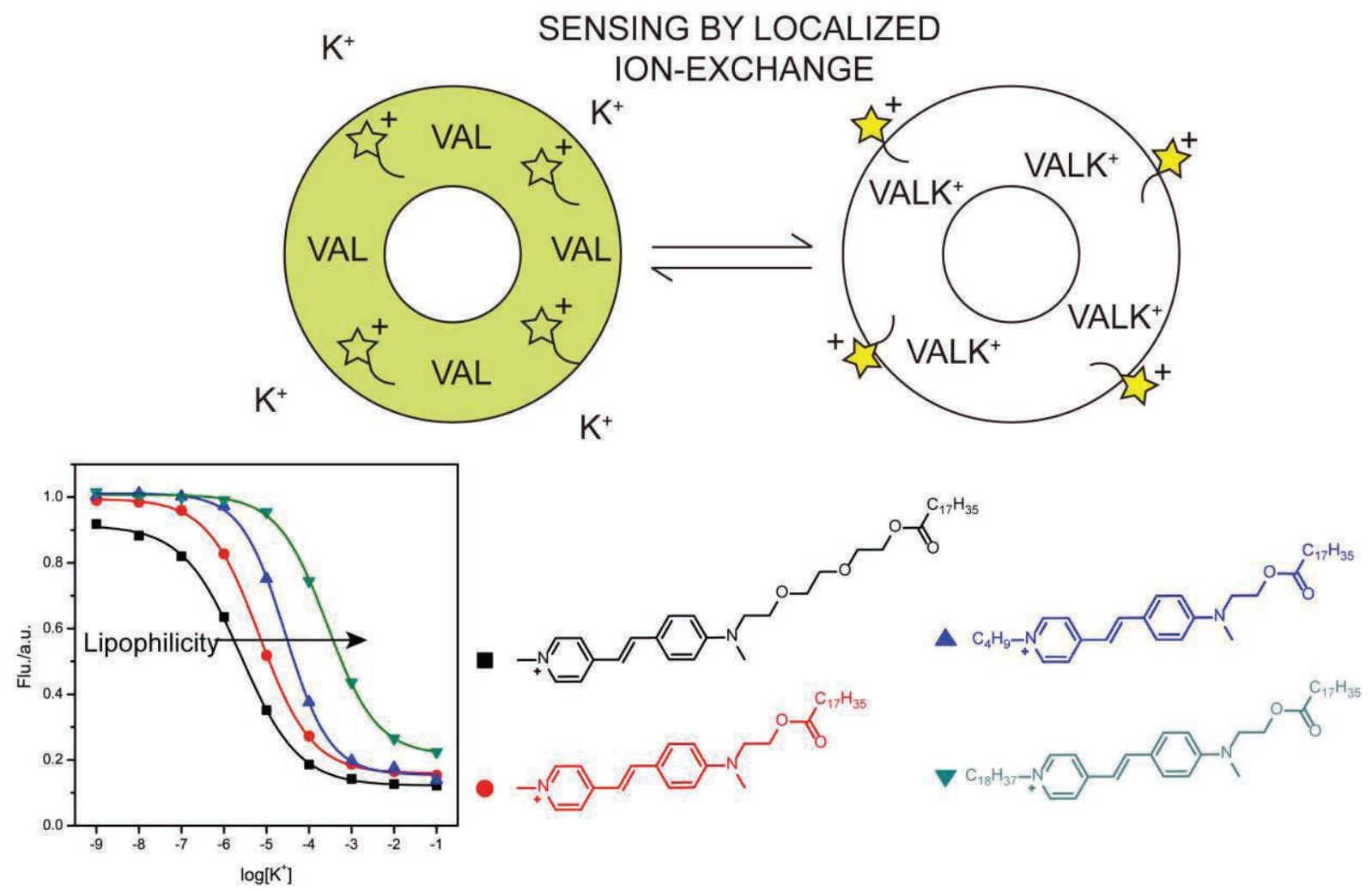

Scheme 1. Ion-exchange mechanism and fluorescence responses of $K^{+}$-selective PS microspheres with solvatochromic dye transducers of different lipophilicity.

\section{References}

[1] X. J. Xie and E. Bakker, Anal Bioanal Chem 2015, 407, 3899-3910.

[2] a) L. Wang, X. J. Xie, J. Y. Zhai and E. Bakker, Chem. Commun. 2016, 52, 14254-14257; b) X. J.
Xie, I. Szilagyi, J. Y. Zhai, L. Wang and E. Bakker, ACS Sens. 2016, 1, 516-520.

[3] X. J. Xie, G. A. Crespo, J. Y. Zhai, I. Szilagyi and E. Bakker, Chem. Commun. 2014, 50, 4592-4595. 